\title{
A novel 3D culture model of tauopathy shows promise as a screening tool for Alzheimer disease therapies
}

Researchers in Germany have developed a 3D model of tauopathy that recapitulates pathological processes observed in neurons of patients with tauopathies such as Alzheimer disease (AD), and could provide an efficient system to test the efficacy of therapies for these disorders.

Diana Seidel and colleagues previously developed a system to monitor realtime neurodegenerative processes in $2 \mathrm{D}$ neuronal cultures. "Since chronic neurodegenerative pathologies like AD require a more complex model system, and to overcome the limitations of 2D models, we established our 3D model," says Seidel.

The researchers transfected enhanced green fluorescent protein (EGFP)-fused tau protein-wild-type, single-mutated or fourfold-mutated-into SH-SY5Y cells (a human neuroblastoma cell line). Tautransfected cells were then differentiated using spheroid-like cultures in a shakerbased system. "Using 3D instead of $2 \mathrm{D}$ cultures, an increased neuronal
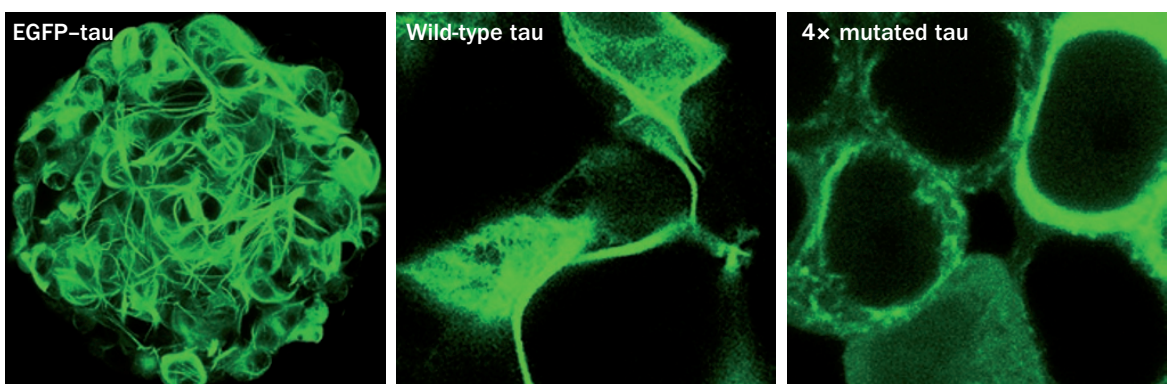

Differentiated SH-SY5Y cells transfected with EGFP-fused tau protein (green) reveals neuron-like morphology. Compared with wild-type tau, fourfold-mutant tau caused enhanced pathology in the 3D cultures. Images courtesy of H.-G. Jahnke. differentiation was observed that was independent of artificial differentiation enhancers," explains Seidel.

A novel microcavity array-based impedance spectroscopy system for the $3 \mathrm{D}$ cultures enabled quantification of the effects of tau mutation number on pathology, with the greatest degeneration being observed in cells expressing fourfoldmutant tau. Furthermore, regenerative effects of enhancers of tau degradation could be observed in real time.

Seidel and colleagues plan to use their model to investigate known inducers of tau pathology and, in collaboration with pharmaceutical companies, to test drugs for tauopathies such as AD.

Katy Malpass

Original article Seidel, D. et al. Induced tauopathy in a novel 3D-culture model mediates neurodegenerative processes: a real-time study on biochips. PLOS ONE 7, e49150 (2012) 